\title{
Bem-estar no ambiente de trabalho em escolas de enfermagem brasileiras
}

\author{
Bienestar en el ambiente laboral en escuelas de enfermería brasileras \\ Wellbeing in the working environment in Brazilian nursing schools
}

\author{
Maria Lúcia do Carmo Cruz Robazzi* \\ Sergio Valverde Marques dos Santos** \\ Rita de Cássia de Marchi Barcellos Dalri ${ }^{* * *}$ \\ Vanessa Augusto Bardaquim ${ }^{* * * *}$ \\ Joab Jefferson da Silva Xavier ${ }^{* * * * *}$ \\ Luiz Almeida da Silva ${ }^{* * * * * *}$ \\ Fabiana Cristina Taubert de Freita ${ }^{* * * * * * *}$ \\ Emiliane Moreno Vichnewski $i^{* * * * * * *}$
}

\section{Autor de correspondencia}

* Enfermeira, Professora Titular da Escola de Enfermagem de Ribeirão Preto, Universidade de São Paulo, E-mail: avrmlccr@usp.br , Ribeirão Preto, São Paulo, Brasil.

** Enfermeiro, Mestre, Doutorando em Ciências pela Escola de Enfermagem de Ribeirão Preto, Universidade de São Paulo. E-mail: sergiovalverdemarques@hotmail.com, Ribeirão Preto, São Paulo, Brasil.

**** Enfermeira, Pós-Doutora pela Escola de Enfermagem de Ribeirão Preto - Universidade de São Paulo. E-mail: ritacmbdalri@bol.com.br, Ribeirão Preto, São Paulo, Brasil.

***** Enfermeira, Doutoranda da Escola de Enfermagem de Ribeirão Preto, Universidade de São Paulo. E-mail: va.bardaquim@usp.br, Ribeirão Preto, São Paulo, Brasil.

****** Doutor pela Escola de Enfermagem de Ribeirão Preto, Universidade de São Paulo. E-mail: joab@ eerp.usp.br, Ribeirão Preto, São Paulo, Brasil.

******** Enfermeiro, Professor Adjunto da Universidade Federal de Goiás - UFG, E-mail: enferluiz@ yahoo.com.br, Catalão, Goiás, Brasil.

******** Fisioterapeuta, Doutorada, Professora da Universidade Ribeirão Preto - UNIP. E-mail: fabi. taubert@hotmail.com, Ribeirão Preto, São Paulo, Brasil.

********** Enfermeira, Mestranda da Escola de Enfermagem de Ribeirão Preto - Universidade de São Paulo. E-mail: emilianemv@gmail.com, Ribeirão Preto, São Paulo, Brasil.

Este es un artículo bajo la

licencia CC BY

(https://creativecommons.org/

licenses/by/4.0/) @) (1)

\section{Resumo}

Objetivo: Avaliar o bem-estar no trabalho de docentes e técnico-administrativos em Escolas de Enfermagem de Universidades públicas brasileiras. Materiais e Método: Estudo descritivo, transversal, quantitativo, com 69 participantes de cinco instituições universitárias. A coleta dos dados ocorreu em 2015-2016. Utilizou-se um questionário semiestruturado para caracterização da população além da Escala de Bem-Estar no Trabalho, Escala de Suporte Organizacional Percebido e Escala de Oportunidades do Trabalho. Para a análise estatística descritiva utilizou-se o software Statistical Package for the Social Science versão 17.0, sendo os dados apresentados por meio de valores absolutos e percentuais. Resultados: Os participantes foram, majoritariamente, do sexo feminino, com 18 a 29 anos, solteiros e com renda mensal de 453,87 a 907,14 dólares. Com relação à categoria profissional, $69,5 \%$ eram técnico-administrativos e $30,5 \%$ docentes. A maioria dos entrevistados trabalhava até 40 horas semanais e $50 \%$ possuíam outro vínculo empregatício. Avaliando o bemestar laboral identificou-se que a maioria dos trabalhadores estava moderadamente alegre, entusiasmada e desenvolvia habilidades importantes. Porém, o trabalho também deixou parte dos trabalhadores irritados, chateados e impacientes. Ao avaliar o suporte organizacional constatou-se que 55,9\% referiram que a Instituição mantém coerência entre diretrizes, metas e ações. Com relação às oportunidades no trabalho a maioria respondeu executar atividades que os agrada. Conclusão: Faz-se necessário oferecer apoio ao desenvolvimento pessoal e laboral dos docentes e trabalhadores técnico-administrativos, contribuindo para o bem-estar no trabalho do servidor público de universidades brasileiras.

Palabras-chave: Qualidade de Vida, Docentes, Trabalhadores, Enfermagem, Condições de trabalho.

Para citar este artículo/ To reference this article/ Para citar este artigo/

Robazzi ML, Santos SV, Darli RC, Bardaquim VA, Xavier JJ, Silva LA, et al. Bem-estar no ambiente de trabalho em escolas de enfermagem brasileiras. Rev. cienc. cuidad. 2019; 16(2):8-20. 


\title{
Resumen
}

Objetivo: Evaluar el bienestar laboral del personal docente y técnico-administrativo en escuelas de enfermería de universidades públicas brasileiras. Materiales y métodos: Estudio descriptivo, transversal, cuantitativo con 69 participantes de cinco instituciones universitarias. La recolección de los datos se realizó en 2015-2016. Se empleó un cuestionario semiestructurado para la caracterización de la población, así como la escala de bienestar en el trabajo, la escala de soporte organizacional percibido y la escala de oportunidades de trabajo. Para el análisis descriptivo se utilizó el software SPSS 17.0, presentando los datos en valores absolutos y relativos. Resultados: Los participantes fueron predominantemente del sexo femenino, con edades entre 18 y 29 años, solteros y con una renta mensual entre U\$ 453.87 a 907.14. En el tipo de vínculo con la institución, $69.5 \%$ fueron técnico-administrativos y $30.5 \%$ docentes. La mayoría de los entrevistados trabaja más de 40 horas a la semana y $50 \%$ tienen otro vínculo laboral. Al evaluar el bienestar laboral se identificó que la mayoría de los trabajadores estaba moderadamente alegre, entusiasmado y desarrollaba habilidades importantes. Sin embargo, también los deja molestos e impacientes. Al evaluarse el soporte organizacional se identificó que $55.9 \%$ refieren que la institución mantiene coherencia entre las directrices, metas y acciones que desarrollan. Relacionado con las oportunidades en el trabajo, la mayoría de los encuestados informaron que realiza actividades que les agradan. Conclusión: Es necesario ofrecer apoyo al desarrollo personal y laboral de los docentes y trabajadores técnico-administrativos, contribuyendo para el bienestar laboral del servidor público de las escuelas de enfermería de universidades brasileiras.

Palabras clave: Calidad de vida, docentes, programas de graduación en enfermería, trabajadores, condiciones de trabajo.

\begin{abstract}
Objective: Evaluate the wellbeing at work of teaching and technical-administrative staff in nursing schools of public Brazilian universities. Materials and methods: Descriptive, cross-sectional, quantitative study with 69 participants from 5 university institutions. The data collection was made in 20152016. A semi-structured questionnaire was used for the characterization of the population, as well as a wellbeing at work scale, the organizational support scale and the job opportunities scale. For the descriptive analysis the software SPSS 17.0 was used, presenting data in absolute and relative values. Results: The participants were predominantly females, ranging ages between 18 and 29, single and with a monthly income between USD $\$ 453.87$ and $\$ 907.14$. For the type of employment function with the institution, $69.5 \%$ were technical-administrative and 30.5\% teaching staff. Most of the interviewed work more than 40 hours per week and 50\% have another employment function. When evaluating the wellbeing at work, it was identified that most of the workers are modestly happy, enthusiastic and developed important skills. However, work also makes them annoyed and impatient. When evaluating organizational support, $55.9 \%$ refer that the institution maintains coherence among the guidelines, goals and actions developed. Relating to job opportunities, most of the interviewed informed performing activities they please. Conclusion: It is necessary to offer support for the personal and labor development of the teaching and the technical-administrative staff, thus contributing for the wellbeing at work of civil servants at nursing schools in public universities in Brazil.
\end{abstract}

Keywords: Life quality, teachers, nursing graduate programs, workers, working conditions.

\section{Introdução}

Nas últimas décadas o ambiente laboral vem sofrendo mudanças consideráveis, até mesmo no âmbito da educação superior, como redução de pessoal, contratos temporários, longas jornadas de trabalho, entre outros. O interesse pelo bem-estar no ambiente do trabalho é devido a estas recentes transformações, que as organizações e os setores de recursos humanos vêm sofrendo (1).
O conceito de bem-estar no contexto do trabalho consiste em acontecimentos relacionados às experiências positivas dos trabalhadores nas organizações (2).

Partindo desse pressuposto, compreende-se que o trabalho do docente universitário no Brasil é desgastante, permeado pelas extensas jornadas de trabalho (3) e as demais atribuições que lhe são destinadas. Os professores universitários brasileiros precisam lidar com as novas tecnologias de ensino-aprendizagem, 
ISSN-PRINT

1794-9831

E-ISSN 2322-7028

Vol. 16 No. 2

May - Ago 2019

Cúcuta, Colombia com as cobranças por alta produtividade acadêmica, o aprimoramento intelectual, a qualidade na educação e ainda colaborar para o melhor ranking no posicionamento das Instituições de Educação Superior (4). Ao se tratar de Escolas de Enfermagem, estes docentes ainda acompanham estágios em Instituições hospitalares.

O financiamento para o ensino universitário e para as pesquisas são limitados, devido aos restritos recursos financeiros e ao reduzido número de professores para compor as vagas existentes em algumas universidades brasileiras, o que acaba sobrecarregando os docentes já efetivos. O produtivismo acadêmico, a precariedade subjetiva e o desgaste mental, levam ao sofrimento psíquico e ao adoecimento dos professores universitários (5).

A outra classe de trabalhadores que também sofre com a sobrecarga de trabalho é a dos técnico-administrativos das universidades públicas brasileiras (6) considerando-se que tal sobrecarga, muitas vezes, pode provocar algum adoecimento mental. Dentre as servidoras técnico-administrativas estudadas em um Instituto Federal de Educação Tecnológica brasileiro, 76,3\% apresentaram algum nível de estresse (7). Ademais, fica claro que há uma limitação dos trabalhadores técnico-administrativos frente ao domínio dos docentes na gestão das universidades (8). Outra questão identificada em um estudo desenvolvido em uma universidade brasileira é o assédio moral no trabalho sofrido tanto pelos docentes como pelos técnico-administrativos (9).

Diante ao exposto, observa-se que os trabalhadores que atuam nas universidades, tanto os professores como os técnico-administrativos podem estar expostos aos diversos fatores de adoecimento. Desta forma, justifica-se a realização deste estudo, uma vez que ao avaliar o bem-estar no trabalho de docentes e de técnico-administrativos de Escolas de Enfermagem de universidades públicas brasileiras, os resultados poderão subsidiar um maior conhecimento sobre o bem-estar desses trabalhadores no ambiente laboral.

\section{Objetivo Geral}

Avaliar o bem-estar no trabalho de docentes e de técnico-administrativos de Escolas de Enfermagem de universidades públicas brasileiras.

\section{Objetivos Específicos}

- Avaliar os dados de caracterização da população;

- Verificar o bem-estar no trabalho dos profissionais;

- Avaliar o suporte organizacional percebido pelos trabalhadores;

- Verificar o efeito de interação das oportunidades no trabalho com os valores pessoais dos profissionais.

\section{Materiais e Método}

Trata-se de um estudo descritivo, transversal e de abordagem quantitativa, desenvolvido em Escolas de Enfermagem de seis universidades públicas brasileiras, sendo duas estaduais e as demais federais, localizadas nos estados de São Paulo, Piauí, Alagoas, Minas Gerais, Goiás e Paraná, cada uma em um estado, conforme representado na Figura 1. Estas instituições de Enfermagem possuem 436 trabalhadores, entre docentes e técnico-administrativos e em cada uma existia um coordenador e responsável pelo estudo. 


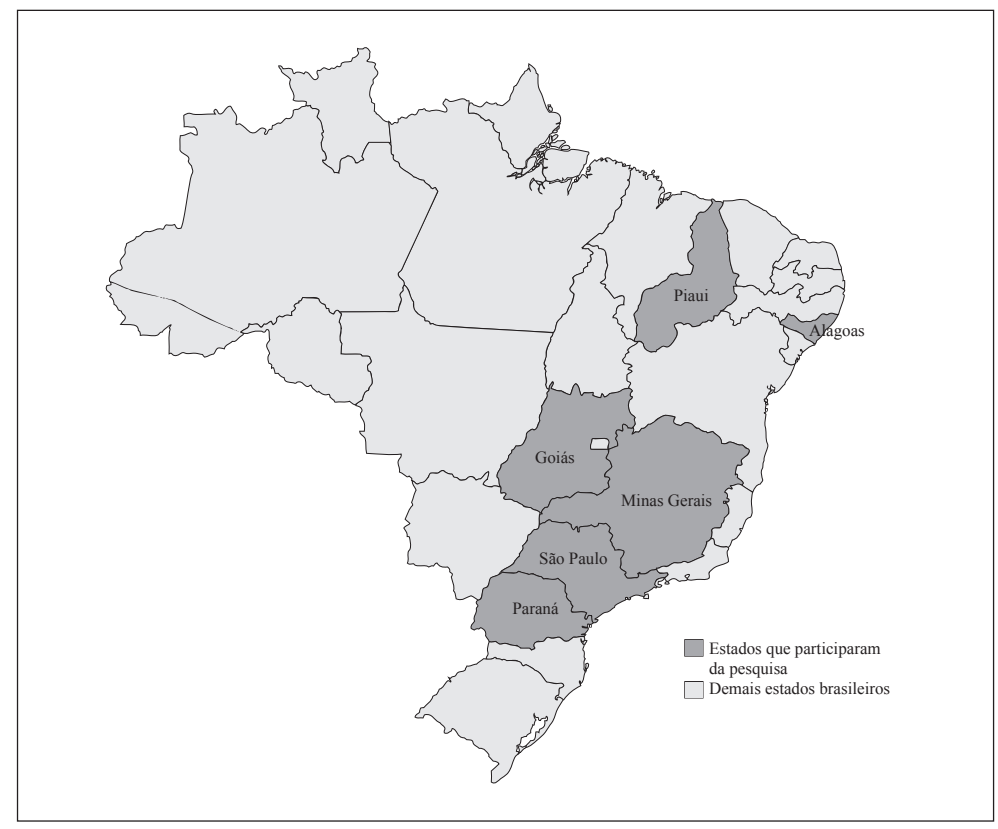

E-ISSN 2322-7028

Vol. 16 No. 2

May - Ago 2019

Cúcuta, Colombia

Figura 1. Localização geográfica dos Estados brasileiros que participaram da pesquisa.

\section{Fonte: IBGE}

Antes de iniciar-se a coleta de dados, os coordenadores de cada uma das universidades e alguns docentes escolhidos por eles receberam treinamento de como proceder à coleta de dados na instituição sede e organizadora do estudo.

Foram adotados os seguintes critérios de inclusão: ser membro ativo e efetivo das Escolas de Enfermagem exercendo a docência ou o trabalho técnico-administrativo nos diversos setores existentes e possuir tempo institucional superior a um ano.

Dos 436 trabalhadores, apenas 69 cumpriram os critérios de inclusão e que aceitaram participar da pesquisa. Assim, o nível de confiança foi de $95 \%$, erro padrão de 0,50 , com $50 \%$ de probabilidade de seleção para todos os participantes. O tipo de amostragem foi aleatório e por conveniência. Deste modo, foram selecionados 11 participantes por estado, exceto São Paulo, que obteve uma amostra de 14 participantes, devido ao tamanho da sua população e número de voluntários que aceitaram participar do estudo.

A coleta dos dados foi realizada nas próprias Escolas de Enfermagem, em horários estabelecidos pela coordenação durante os anos de 2015 e 2016. Todos os participantes receberam envelopes fechados, contendo os instrumentos autoaplicáveis e o Termo de Con- sentimento Livre e Esclarecido, sendo-lhes ofertada uma breve apresentação da pesquisa.

Foram utilizados quatro instrumentos. O primeiro foi um questionário semiestruturado com 19 questões, com intuito de avaliar dados de caracterização da população, contendo variáveis sócio demográficas e laborais. Este instrumento foi elaborado pelos autores, com base na literatura. Cabe mencionar que este questionário passou por processo de refinamento com juízes especialistas na área de conhecimento e, posteriormente, por teste piloto, para sua validação.

O segundo instrumento utilizado foi a Escala de Bem-Estar no Trabalho de Paschoal. Esta escala é do tipo likert, composta por 30 questões e abrange duas partes, uma de afeto e a outra de expressão/realização no trabalho. Foi construída e validada no Brasil por Paschoal e Tamoyo, com nível de fidedignidade de 0,93. A sua versão final ficou composta por três fatores: afeto positivo com nove itens; afeto negativo com 12 itens e o fator realização com nove itens (10).

O terceiro instrumento utilizado foi a Escala de Suporte Organizacional Percebido, também construída e validada no Brasil por Tamoyo e colaboradores, com $\alpha$ superior a $0,80 \mathrm{em}$ todos os seus itens. É composta por 42 itens divididos em seis fatores: gestão organi- 
ISSN-PRINT

1794-9831

E-ISSN 2322-7028

Vol. 16 No. 2

May - Ago 2019

Cúcuta, Colombia zacional do desempenho, sobrecarga de trabalho, percepção de suporte material, política de pagamento e desenvolvimento, estilo da gestão da chefia e suporte social do grupo (11).

O quarto instrumento utilizado foi a Escala de Oportunidades do Trabalho, desenvolvida para permitir a avaliação do efeito de interação das oportunidades no trabalho com os valores pessoais; também foi construída e validada no Brasil por Paschoal e Tamoyo, com nível de confiabilidade superior a de $0,90 \mathrm{em}$ todos os itens. Possui 20 itens, que avaliam as oportunidades de abertura a mudanças, de autopromoção, de autotranscedência e de oportunidades de conservação (10).

Para a análise estatística descritiva foi utilizado o software Statistical Package for the Social Science versão 17.0. Os dados foram apresentados por meio de valores absolutos, percentuais e de forma descritiva.

Com base na Resolução 466 de 2012, este estudo foi aprovado por Comitê de Ética em Pesquisa da Escola de Enfermagem de Ribeirão Preto, da Universidade de São Paulo, conforme Parecer n ${ }^{\circ}$ 1.196.462.

\section{Resultados}

A amostra foi composta em sua maioria por participantes do sexo feminino $(83,6 \%)$, com idade en- tre 18 e 29 anos $(67,6 \%)$, solteira $(68,9 \%)$, da cor branca $(46,7 \%)$, com ensino superior incompleto $(59,2 \%)$ e com renda mensal de 453,87 a 907,14 dólares (22,5\%), valor cotado em 5 de janeiro de 2018 . Parte dos participantes possuía quatro ou mais filhos (48,3\%), 75,1\% tinham moradia própria e 47,4\% utilizavam o ônibus como meio de locomoção para a universidade.

Com relação à categoria profissional, 48 trabalhadores $(69,5 \%)$ eram técnico-administrativos e 21 (30,5\%) docentes das Escolas de Enfermagem das universidades estudadas. Verificou-se que o tempo de atuação institucional prevalente foi de até 10 anos $(57,6 \%)$ para os técnico-administrativos, e entre 10 e 28 anos para os docentes $(51,5 \%)$. Com relação ao turno de trabalho, entre os docentes, a maior parte atuava no período manhã/tarde $(44,4 \%)$, enquanto que entre os técnico-administrativos, a maioria atuava no período da manhã e tarde (69\%). Referente a carga horária de trabalho, $89 \%$ dos técnicos tinham uma carga de 40 horas semanais de trabalho, já os docentes, apenas $33 \%$ declaram trabalhar 40 horas semanais, enquanto os demais relataram possuir carga horária entre $44 \mathrm{e}$ 60 horas.

A Tabela 1 apresenta a avaliação do bem-estar no trabalho, segundo itens de afeto, de acordo com a Escala de Paschoal, 2008. 
Maria Lúcia do Carmo Cruz Robazzi, Sergio Valverde Marques dos Santos, Rita de Cássia de Marchi Barcellos Dalri, Vanessa Augusto Bardaquim, Joab Jefferson da Silva Xavier, Luiz Almeida da Silva, Fabiana Cristina Taubert de Freita, Emiliane Moreno Vichnewski

Tabela 1. Avaliação do Bem-estar no Trabalho dos docentes e técnico-administrativos, segundo itens de afeto, de acordo com a Escala de Paschoal (2008), 2015-2016, (n=66)*

\begin{tabular}{|c|c|c|c|c|c|c|c|c|c|c|}
\hline \multirow{2}{*}{$\begin{array}{l}\text { Nos últimos seis meses meu trabalho } \\
\text { tem me deixado: }\end{array}$} & \multicolumn{2}{|c|}{ Nem um pouco } & \multicolumn{2}{|c|}{ Um pouco } & \multicolumn{2}{|c|}{ Moderadamente } & \multicolumn{2}{|c|}{ Bastante } & \multicolumn{2}{|c|}{ Extremamente } \\
\hline & f & $\%$ & f & $\%$ & f & $\%$ & f & $\%$ & f & $\%$ \\
\hline Alegre & 7 & 10,6 & 9 & 13,6 & 34 & 51,5 & 12 & 18,2 & 4 & 6,1 \\
\hline Preocupado & 10 & 15,2 & 21 & 31,8 & 14 & 21,2 & 12 & 18,2 & 9 & 13,6 \\
\hline Disposto & 7 & 10,6 & 13 & 19,7 & 27 & 40,9 & 16 & 24,2 & 3 & 4,5 \\
\hline Contente & 7 & 10,6 & 13 & 19,7 & 31 & 47,0 & 12 & 18,2 & 3 & 4,5 \\
\hline Irritado & 15 & 22,7 & 30 & 45,5 & 16 & 24,2 & 2 & 3,0 & 3 & 4,5 \\
\hline Deprimido & 41 & 62,1 & 14 & 21,2 & 7 & 10,6 & 4 & 6,1 & - & - \\
\hline Entediado & 38 & 57,6 & 21 & 31,8 & 4 & 6,1 & 2 & 3,0 & 1 & 1,5 \\
\hline Animado & 5 & 7,6 & 13 & 19,7 & 27 & 40,9 & 20 & 30,3 & 1 & 1,5 \\
\hline Chateado & 19 & 28,8 & 29 & 43,8 & 11 & 16,7 & 3 & 4,5 & 4 & 6,1 \\
\hline Impaciente & 23 & 34,8 & 25 & 37,9 & 13 & 19,7 & 4 & 6,1 & 1 & 1,5 \\
\hline Entusiasmado & 12 & 18,2 & 9 & 13,6 & 32 & 48,4 & 9 & 13,6 & 4 & 6,1 \\
\hline Ansioso & 16 & 24,2 & 17 & 25,8 & 19 & 28,8 & 9 & 13,6 & 5 & 7,6 \\
\hline Feliz & 6 & 9,1 & 14 & 21,2 & 24 & 36,4 & 20 & 30,3 & 2 & 3,0 \\
\hline Frustrado & 34 & 51,5 & 15 & 22,7 & 6 & 9,1 & 7 & 10,6 & 4 & 6,1 \\
\hline Incomodado & 25 & 37,9 & 21 & 31,8 & 8 & 12,1 & 8 & 12,1 & 4 & 6,1 \\
\hline Nervoso & 31 & 47,0 & 16 & 24,2 & 10 & 15,2 & 6 & 9,1 & 3 & 4,5 \\
\hline Empolgado & 14 & 21,5 & 8 & 12,3 & 28 & 43,1 & 9 & 13,8 & 6 & 9,2 \\
\hline Tenso & 20 & 30,3 & 24 & 36,4 & 10 & 15,2 & 6 & 9,1 & 6 & 9,1 \\
\hline Orgulhoso & 9 & 13,6 & 10 & 15,2 & 26 & 39,4 & 13 & 19,7 & 8 & 12,1 \\
\hline Com raiva & 37 & 56,1 & 20 & 30,3 & 5 & 7,6 & 4 & 6,1 & - & - \\
\hline Tranquilo & 11 & 16,7 & 20 & 30,3 & 21 & 31,8 & 7 & 10,6 & 7 & 10,6 \\
\hline
\end{tabular}

*Foram excluídos três instrumentos, devido aos erros de preenchimento

Com relação ao bem-estar no trabalho foram verificados os itens de afeto, relacionados às emoções e aos humores positivos e negativos dos docentes e técnico-administrativos no trabalho e percebeu-se que, o trabalho nos últimos seis meses deixou os trabalhadores moderadamente: alegres $(26,4 \%$ docentes, $25,1 \%$ técnico-administrativos); contentes $(25,8 \%$ docentes, $21,2 \%$ técnico-administrativos); entusiasmados (31,2\% docentes, $17,2 \%$ técnico-administrativos) e empolgados $(21,4 \%$ docentes, $21,7 \%$ técnico-admi- nistrativos). Entretanto, o trabalho também deixou parte dos trabalhadores um pouco: irritados (23,3\% docentes, $22,2 \%$ técnico-administrativos); chateados ( $26,6 \%$ docentes, $17,2 \%$ técnico-administrativos) e impacientes $(10,6 \%$ docentes, $27,3 \%$ técnico-administrativos), conforme apresentado na Tabela 1 .

A Tabela 2 apresenta a avaliação do bem-estar no trabalho, segundo itens de expressividade/realização no trabalho. 
ISSN-PRINT

$1794-9831$

E-ISSN 2322-7028

Vol. 16 No. 2

May - Ago 2019

Cúcuta, Colombia

Tabela 2. Avaliação do Bem-estar no Trabalho dos docentes e técnico-administrativos, segundo itens de expressividade/ realização no trabalho de acordo com a Escala de Paschoal (2008). 2015-2016, (n=66)*

\begin{tabular}{|c|c|c|c|c|c|c|c|c|c|c|}
\hline \multirow{2}{*}{ Neste trabalho: } & \multicolumn{2}{|c|}{$\begin{array}{l}\text { Discordo } \\
\text { totalmente }\end{array}$} & \multicolumn{2}{|c|}{ Discordo } & \multicolumn{2}{|c|}{$\begin{array}{c}\text { Concordo em } \\
\text { parte }\end{array}$} & \multicolumn{2}{|c|}{ Concordo } & \multicolumn{2}{|c|}{$\begin{array}{l}\text { Concordo } \\
\text { totalmente }\end{array}$} \\
\hline & f & $\%$ & f & $\%$ & f & $\%$ & f & $\%$ & f & $\%$ \\
\hline Realizo meu potencial & 1 & 1,5 & 1 & 1,5 & 20 & 30,3 & 20 & 30,3 & 24 & 36,4 \\
\hline Desenvolvo habilidades que considero importantes & 1 & 1,5 & - & - & 3 & 4,5 & 31 & 47,0 & 31 & 47,0 \\
\hline Realizo atividades que expressam minhas capacidades & 1 & 1,5 & - & - & 15 & 22,7 & 28 & 42,4 & 22 & 33,3 \\
\hline Consigo recompensas importantes para mim & 3 & 4,5 & 4 & 6,1 & 27 & 40,9 & 18 & 27,3 & 14 & 21,2 \\
\hline Supero desafios & 1 & 1,5 & - & - & 20 & 30,3 & 28 & 42,4 & 17 & 25,8 \\
\hline Atinjo resultados que valorizo & 1 & 1,5 & - & - & 15 & 22,7 & 30 & 45,5 & 20 & 30,3 \\
\hline Avanço nas metas que estabeleci para minha vida & 2 & 1,5 & 2 & 3,0 & 16 & 24,2 & 29 & 43,9 & 18 & 27,3 \\
\hline Faço o que realmente gosto de fazer & 1 & 1,5 & - & - & 14 & 21,2 & 21 & 31,8 & 30 & 45,5 \\
\hline Expresso o que há de melhor em mim & 1 & 1,5 & 1 & 1,5 & 16 & 24,2 & 18 & 27,3 & 30 & 45,5 \\
\hline
\end{tabular}

*Foram excluídos três instrumentos, devido erro de preenchimento

Ao observar os itens de expressividade/realização no trabalho, que diz respeito à percepção dos trabalhadores com relação ao desenvolvimento de suas habilidades e potencial no trabalho, além do avanço no alcance de suas metas de vida, notou-se que parte deles concordaram que: desenvolvia habilidades importantes $(12,9 \%$ docentes, $34,1 \%$ técnico-administrativos); atingiam resultados que valorizavam (26,6\% docentes, 18,9\% técnico-administrativos); e, avançavam nas metas que estabeleceram para a vida $(17,2 \%$ docentes, 26,7\% técnico-administrativos). Além disso, alguns concordaram, em parte, que conseguiam recompensas importantes $(29,6 \%$ docentes, $11,3 \%$ técnico-administrativos); e, faziam o que realmente gostavam ( $6,0 \%$ docentes, $15,2 \%$ técnico-administrativos), conforme apresentado na Tabela 2.

A Tabela 3 apresenta a avaliação do suporte organizacional percebido pelos participantes das instituições.

Tabela 3. Avaliação do Suporte Organizacional Percebido pelos docentes e técnico-administrativos de acordo com a Escala de Suporte Organizacional Percebido. 2015-2016, $(n=69)$

\begin{tabular}{|c|c|c|c|c|c|c|c|c|c|c|}
\hline \multirow{2}{*}{ Fatores } & \multicolumn{2}{|c|}{ Nunca } & \multicolumn{2}{|c|}{ Raramente } & \multicolumn{2}{|c|}{ Algumas vezes } & \multicolumn{2}{|c|}{ Frequentemente } & \multicolumn{2}{|c|}{ Sempre } \\
\hline & F.M* & $\%$ & F. $\mathbf{M}$ & $\%$ & F. $\mathbf{M}$ & $\%$ & F. $\mathbf{M}$ & $\%$ & F. $\mathbf{M}$ & $\%$ \\
\hline Suporte material & 8,1 & 11,7 & 12,7 & 18,4 & 26 & 37,6 & 19,5 & 28,2 & 6,1 & 8,8 \\
\hline Gestão de Desempenho & 10,5 & 15,2 & 11,4 & 16,5 & 22,8 & 33 & 19,8 & 28,6 & 5,7 & 8,2 \\
\hline Carga de trabalho & 12,7 & 18,4 & 12,5 & 18,1 & 22,1 & 32 & 15,3 & 22,1 & 5,8 & 8,4 \\
\hline Apoio Social & 2,1 & 3 & 8,7 & 12,6 & 23,5 & 34 & 23,1 & 33,4 & 12,1 & 17,5 \\
\hline Ascensão e salários & 8,2 & 11,8 & 13,8 & 20 & 23,4 & 33,9 & 16 & 23,1 & 7,6 & 11 \\
\hline
\end{tabular}

* Frequência Média das afirmativas conforme respostas dos participantes

Ao avaliar o suporte organizacional percebido pelos docentes e técnico-administrativos, que foi medido pelos fatores de gestão organizacional do desempenho, sobrecarga de trabalho, percepção de suporte material, política de pagamento e desenvolvimento, estilo de gestão da chefia e suporte social do grupo, observou-se que das respostas às afirmativas, os participantes responderam que "algumas vezes": a
Instituição proporcionava suporte material adequado (13\% docentes, $24,6 \%$ técnico-administrativos); possuía uma gestão de desempenho de qualidade (7,5\% docentes, $25,5 \%$ técnico-administrativos); tinha uma sobrecarga de trabalho $(17,3 \%$ docentes, $14,7 \%$ técnico-administrativos); possuía apoio social por parte da equipe e da Instituição (18,3\% docentes, $13,7 \%$ técnico-administrativos); e, tinha 
Maria Lúcia do Carmo Cruz Robazzi, Sergio Valverde Marques dos Santos, Rita de Cássia de Marchi Barcellos Dalri, Vanessa Augusto Bardaquim, Joab Jefferson da Silva Xavier, Luiz Almeida da Silva, Fabiana Cristina Taubert de Freita, Emiliane Moreno Vichnewski

ascensão no trabalho e salários adequado (10,2\% docentes, $23,7 \%$ técnico-administrativos), de acordo com a Tabela 3.
Na Tabela 4 é apresentada a avaliação das oportunidades no trabalho dos participantes das instituições.

Tabela 4. Avaliação das Oportunidades no trabalho dos docentes e técnico-administrativos de acordo com a Escala de Oportunidades do Trabalho. 2015-2016, $(\mathrm{n}=68) *$

\begin{tabular}{|c|c|c|c|c|c|c|c|c|}
\hline \multirow{2}{*}{ Neste trabalho, tenho oportunidade de: } & \multicolumn{2}{|c|}{ Nenhuma } & \multicolumn{2}{|c|}{ Pouca } & \multicolumn{2}{|c|}{ Moderada } & \multicolumn{2}{|c|}{ Muita } \\
\hline & f & $\%$ & $\mathbf{f}$ & $\%$ & $\mathbf{f}$ & $\%$ & f & $\%$ \\
\hline Exercer minha criatividade & 2 & 2,9 & 4 & 5,9 & 34 & 50,0 & 28 & 41,2 \\
\hline Ter prestígio & - & - & 12 & 17,6 & 34 & 50,0 & 22 & 32,4 \\
\hline Tratar as pessoas com igualdade & 2 & 2,9 & 3 & 4,4 & 19 & 27,9 & 44 & 64,7 \\
\hline Mostrar minhas habilidades & 2 & 2,9 & 2 & 2,9 & 34 & 50,0 & 30 & 44,1 \\
\hline Desenvolver minhas tarefas num ambiente seguro & - & - & 9 & 13,2 & 29 & 42,6 & 30 & 44,1 \\
\hline Executar tarefas novas & 1 & 1,5 & 9 & 13,2 & 31 & 45,6 & 27 & 39,7 \\
\hline Exercitar meu auto-controle & 1 & 1,5 & 3 & 4,4 & 28 & 41,2 & 36 & 52,9 \\
\hline Considerar diferentes opiniões & 1 & 1,5 & 10 & 14,7 & 26 & 38,2 & 30 & 44,1 \\
\hline Realizar minhas atividades de maneira tradicional & 3 & 4,5 & 10 & 14,9 & 37 & 55,2 & 17 & 25,4 \\
\hline Executar atividades que me agradam & 1 & 1,5 & 5 & 7,4 & 40 & 58,8 & 22 & 32,4 \\
\hline Exercer minha autonomia & 1 & 1,5 & 7 & 10,3 & 36 & 52,9 & 24 & 35,3 \\
\hline Ajudar as pessoas ao meu redor & - & - & 5 & 7,4 & 27 & 39,7 & 36 & 52,9 \\
\hline Ser admirado pelas pessoas & 2 & 2,9 & 9 & 13,2 & 37 & 54,4 & 20 & 29,4 \\
\hline Ter estabilidade & - & - & 7 & 10,3 & 17 & 25,0 & 44 & 64,7 \\
\hline Ter desafios & - & - & 7 & 10,3 & 28 & 41,2 & 33 & 48,5 \\
\hline Influenciar pessoas & 2 & 2,9 & 9 & 13,2 & 31 & 45,6 & 26 & 38,2 \\
\hline Ser leal aos meus colegas & - & - & 3 & 4,4 & 17 & 25,0 & 48 & 70,6 \\
\hline Contribuir para a preservação do meio ambiente & 3 & 4,4 & 9 & 13,2 & 25 & 36,8 & 31 & 45,6 \\
\hline Realizar tarefas prazerosas & - & - & 6 & 8,8 & 34 & 50,0 & 28 & 41,2 \\
\hline Contribuir para preservação de costumes & - & - & 10 & 14,7 & 35 & 51,5 & 23 & 33,8 \\
\hline
\end{tabular}

*Foi excluído um instrumento, devido erro de preenchimento

Foram avaliadas as oportunidades no trabalho dos docentes e técnico-administrativos, que diz respeito às oportunidades de abertura a mudanças, de autopromoção, de autotranscedência e de oportunidades de conservação. Observou-se que a maioria dos trabalhadores concorda moderadamente que no trabalho eles têm oportunidade de: exercer sua criatividade (29,4\% docentes, $20,6 \%$ técnico-administrativos); ter prestígio (11,7\% docentes, 38,3\% técnico-administrativos); mostrar suas habilidades $(29,4 \%$ docentes, $20,6 \%$ técnico-administrativos); realizar suas atividades de maneira tradicional (29,7\% docentes, $25,5 \%$ técnico-administrativos); exercer sua autonomia (27,7\% docentes, $25,2 \%$ técnico-administrativos); ser admirado pelas pessoas (24,3\% docentes, $30,1 \%$ técnico-administrativos); realizar tarefas prazerosas (26,4\% docentes, $23,6 \%$ técnico-administrativos); e, contribuir para preservação de costumes $(22,8 \%$ docentes, $27,4 \%$ técnico-administrativos), conforme Tabela 4.

\section{Discussão}

A educação superior no Brasil sofreu transformações significativas, principalmente a partir do final dos anos 1990. Essas transformações atendem a uma lógica de mercado que modifica a estrutura organizacional universitária, suas funções acadêmicas e o trabalho docente (12). Trata-se de um modelo de gestão pública adotado pelos governo federal e estaduais, no qual prevalecem uma racionalidade organizacional e a avaliação quantitativa do trabalho docente (13) e consequentemente, dos demais trabalhadores das universidades.

Sabe-se que o suporte social e os estilos de vida saudável constituem-se nos alicerces do sucesso acadêmico. Fomentar estilos de vida saudável também tem sido tratado 
ISSN-PRINT

1794-9831

E-ISSN 2322-7028

Vol. 16 No. 2

May - Ago 2019

Cúcuta, Colombia como forma de intervenção no combate às causas do insucesso escolar. Problemas de saúde têm origem em hábitos e estilos de vida próprios, cuja prevenção ou remediação necessita de alterações em atitudes e comportamentos, dependendo, dos fatores pessoais e suas interações com o ambiente físico, social e cultural, da situação econômicofinanceira e das aprendizagens sociais (14).

Neste sentido, faz-se necessário que as Organizações promovam a saúde física, mental e afetiva do trabalhador, considerando que o sofrimento psicológico aumenta o estresse relacionado ao trabalho (6), podendo acarretar em um menor desempenho das suas atividades laborais. Para que haja melhoria da Qualidade de Vida no Trabalho (QVT) nas universidades, devem ser considerados os aspectos relacionados ao salário, as condições do ambiente de trabalho e os planos de carreira (15). Os trabalhadores estão cada vez mais informados e conscientes dos seus direitos e assim exercem um maior poder de cobrança (16).

Com relação ao bem-estar no trabalho, aos itens de afeto relacionados às emoções e humores positivos e negativos e aos itens de expressividade/realização dos docentes e técnico-administrativos, percebeu-se que a maioria estava moderadamente alegres e apenas alguns permaneceram contentes, entusiasmados e empolgados. Entretanto, também estavam irritados, chateados e impacientes com o trabalho. Além disso, somente parte deles sentia que desenvolvia habilidades importantes, atingia resultados que valorizavam e avançava nas metas que estabeleceu para a vida.

As afeições positivas no ambiente laboral, que são determinadas pelas emoções como alegria, felicidade, contentamento, bem como as experiências adequadas e agradáveis vivenciadas no trabalho, muitas vezes, podem estar relacionadas às práticas culturais de atuação. Estas práticas dizem respeito a inovações em ideias, pela busca de melhorias, de investimentos em pesquisas e são vistas como forma de estímulo acadêmico (17).

As dimensões de afeto e realização/expressividade no trabalho, em algumas situações, podem ser capazes de estruturar o bem-estar no trabalho, como por exemplo: ter oportunidade de desenvolver habilidades importantes, atingir resultados que valorizem o trabalhador, avançar nas metas que estabelece para a vida, entre outros. O contexto laboral pode se apresentar como um ambiente cheio de emoções positi- vas, de realização pessoal e profissional, bem como de constituição da felicidade (10).

Ao avaliar o suporte organizacional percebido pelos docentes e técnico-administrativos, observou-se que parte deles referiu que algumas vezes a organização adquire equipamentos modernos, mantém coerência entre diretrizes, metas e ações, ouve a opinião dos trabalhadores na resolução dos problemas e investe em treinamentos. Ao avaliar as oportunidades no trabalho dessas pessoas, notou-se que a maioria concorda moderadamente que o trabalho possui diversas formas de oportunidade, como por exemplo, exercer sua criatividade e ter prestígio, entre outros.

O trabalhador ao perceber que a instituição incentiva sua participação na gestão, passa a valorizar o trabalho e demonstrar uma maior flexibilidade para a ações, bem como tende a apresentar uma hierarquia mais reduzida, estando positivamente associada ao bem-estar organizacional (18).

Entretanto, em algumas situações, os privilégios e oportunidades podem vir acompanhados de sobrecargas de trabalho. Em uma pesquisa realizada com docentes de uma universidade, identificou-se que os participantes possuíam sobrecarga de trabalho. Neste estudo houve relatos de que alguns trabalhadores exerciam suas atividades acima de 40 horas por semana, pois tinham que trabalhar aos finais de semana, exercer atividades na graduação e pós-graduação, participar em comissões universitárias e realizar produção científica (5).

Estas situações podem provocar o adoecimento mental do trabalhador e reduzir o seu nível de bem-estar no trabalho, podendo acarretar a perda da produtividade e do desempenho acadêmico. Docentes universitários da área da saúde perceberam e identificaram situações estressantes relacionadas ao trabalho no contexto universitário (19). Neste contexto, torna-se relevante mencionar que estes docentes, além de exercer suas atividades dentro da universidade, ainda acompanham estágios em Instituições hospitalares e Unidades Básicas de Saúde, acarretando, ainda mais, estresse decorrente do trabalho.

As condições organizacionais quando são desfavoráveis aos trabalhadores, podem colaborar para a elevação dos níveis de estresse ocupacional, devido as situações vivenciadas na execução das tarefas. Estes fatores tendem a afetar o bem-estar organizacional e a saúde do trabalhador, que podem refletir na qualidade e bem-estar no trabalho e em atitudes e afeições negativas para com as 
atividades laborais, com o ambiente de trabalho, com os colegas e também com a Instituição (20).

Quando não há este apoio da organização e um ambiente laboral adequado, os trabalhadores docentes e técnicos-administrativos são expostos a diversas formas de agressão e adoecimento pelo trabalho, que reduz o nível de bem-estar e produtividade. Estudos nacionais e internacionais têm demostrado as diversas formas de agressão que estes trabalhadores têm vivenciado nas Universidades, como: estresse, assédio moral, sobrecarga de trabalho, depressão, pressão, entre outros (21-24).

Estes fatos, somados a falta de oportunidade e autonomia no trabalho, pode gerar diversos fatores que os trabalhadores utilizam como válvula de escape para o desgaste e o adoecimento mental, como o alcoolismo, as drogas, a automedicação, o afastamento do trabalho, as aposentadorias por invalidez, dentre outros fatores (25-28).

O suporte organizacional oferecido pela Instituição está relacionado a uma à gestão de desempenho, que envolve práticas como estabelecimento de metas, valorização de idéias, autonomia, apoio social e modernização das tecnologias (29).

Com relação as oportunidades no trabalho, sabe-se que quanto mais a Instituição promove o trabalhador por meio de oportunidades, de forma que demonstre sua autonomia e habilidades, mais satisfação e bemestar laboral este profissional irá desenvolver. Com isso, o trabalhador ganha em qualidade de vida no trabalho e a empresa em melhor rendimento e aproveitamento deste trabalhador.

Tendo em vista o contexto apresentado, percebese que quanto mais uma organização recompensar, apoiar, reconhecer e tratar de forma adequada seus trabalhadores, proporcionando recompensas condizentes às suas responsabilidades, maior será o seu nível de bem-estar no trabalho (30) e consequentemente ocorrerá maior produtividade e satisfação.

Neste sentido, pode se dizer que, a análise das condições organizacionais possui impacto significativo sobre os diferentes elementos do bem-estar, que pode contribuir no planejamento de diferentes intervenções no ambiente laboral trabalho (29).

Como limitação do estudo, torna-se importante ressaltar que, o fato de ser de corte transversal, avaliado em um único momento no contexto laboral dos participantes, o que impossibilitou outras análises e a generalização dos resultados, impedindo uma discussão mais ampla e aprofundada. Outro aspecto refere-se ao fato da coleta de dados ter sido feita em anos distintos, em decorrência de problemas no âmbito das próprias universidades.

Entretanto, foi possível constatar diversas questões sobre o ambiente laboral dos docentes e técnico-administrativos de algumas universidades brasileiras, o que mostra a sua pouca situação de bem estar.

\section{Conclusão}

- O bem-estar no trabalho está relacionado à valorização, desempenho e qualidade de vida do trabalhador na Instituição. Dessa forma, deve ser repensada a estrutura organizacional das instituições públicas de ensino superior no Brasil, pois muitas vezes, elas não oferecem aos trabalhadores condições adequadas para o desenvolvimento laboral pleno e de qualidade.

- Faz-se necessário providenciar equipamentos modernos, não deixar faltar os materiais necessários, considerar a opinião dos trabalhadores, principalmente dos técnicos-administrativos para resolver os problemas internos, não sobrecarregar alguns servidores e investir em treinamento para todos; dando-lhes oportunidades de realizar tarefas prazerosas e exercer as suas habilidades, criatividade e autonomia, oferecer programas de apoio ao desenvolvimento da carreira, contribuindo, dessa forma, para o bem-estar no trabalho do servidor público, bem como para uma melhor qualidade de vida e felicidade.

- Este fato chama a atenção para a realização de novas investigações, com métodos de avaliação diferenciados, com por exemplo, os estudos experimentais e/ou longitudinais. Isto, no intuito de comparar grupos amostrais diferentes, em busca da causa/efeito do trabalho sobre o trabalhador.

\section{Agradecimentos}

Ao CNPQ, pelo apoio para o desenvolvimento do projeto.

\section{Conflito de interesses}

Os autores declaram não ter nenhum conflito de interesses. 
ISSN-PRINT

$1794-9831$

E-ISSN 2322-7028

Vol. 16 No. 2

May - Ago 2019

Cúcuta, Colombia

\section{Referências}

1. Santos GB, Ceballos AGC. Bem-estar no trabalho: estudo de revisão. Psic Estudo. [Internet] 2013. [Acesso em $12 \mathrm{dez}$ 2017]; 18(2): 247-55. Disponível em: http://www.redalyc.org/articulo. oa? id=287128992006

2. Paschoal T, Demo G, Fogaça N, Ponte V, Edrei L, et al. Bem-estar no trabalho: cenário dos estudos brasileiros publicados na primeira década do novo milênio. Portugal. Tourism \& Management Studies [Internet] 2013. [Acesso em 20 ago 2017]; 2:383-95. Disponível em: http://www.redalyc.org/articulo. oa? id $=388743875002$

3. Oliveira JM, Santos PF, Feliciano RG, Assis MM, Cortez EA, Valente GSC. Riscos e doenças ocupacionais do docente universitário de enfermagem: implicações na saúde do trabalhador. R. Pesq. Cuid Fundam Online. [Internet] 2013. [Acesso em 12 fev 2017]; 5(1):3267-75. Disponível em: http://bases. bireme.br/cgi-bin/wxislind.exe/iah/online/?IsisScript=iah/iah.xis\&src=google\&base=LILACS\&lang $=\mathrm{p} \&$ nextAction $=\operatorname{lnk} \&$ exprSearch $=686267 \&$ indexSearch $=$ ID

4. Mendonça H, Ferreira MC, Caetano A, Torres CV. Cultura Organizacional, Coping e Bem-Estar Subjetivo: um Estudo Com Professores de Universidades Brasileiras. Rev Psicol Organ Trab. [Internet] 2014. [Acesso em 20 abr 2017]; 14(2):230-44. Disponível em: http://pepsic.bvsalud.org/pdf/rpot/v14n2/v14n2a09.pdf

5. Bernardo MH. Produtivismo e precariedade subjetiva na universidade pública: o desgaste mental dos docentes. Psic \& Socied. [Internet] 2014. [Acesso em 20 abr 2017]; 26(n.spe.): 129-39. Disponível em: http://www.redalyc.org/html/3093/309331565014/

6. Vilas Boas AA, Morin EM. Indicadores de qualidade de vida no trabalho para professores de instituições públicas de ensino superior: uma comparação entre Brasil e Canadá. Rev Contextus. [Internet] 2016. [Acesso em 02 Jul 2017]; 14(2):170-98. Disponível em: http://repositorio.ufc.br/ri/bitstream/ riufc/22475/1/2016_art_aavboas.pdf

7. Pego ZO, Soares MG. O estresse ocupacional de servidoras técnico- administrativas. Rev Alcanc. Universidade Vale do Itajaí. [Internet] 2016. [Acesso em 01 out 2017]; 23(2): 156-69. Disponível em: http://www.redalyc.org/articulo.oa?id=477749648004

8. Gonçalves $\mathrm{PB}$, Santos MR, Chaves MR, Silva AV. O contexto histórico das universidades federais no Brasil e a participação de docentes, discentes e técnicos administrativos no processo de tomada de decisão nessas instituições públicas. p.190-97. In: Anais do Simpósio de Metodologias Ativas: Inovações para o ensino e aprendizagem na educação básica e superior [= Blucher Education Proceedings, v. 2, n. 1]. São Paulo: Blucher, [Internet] 2017. [Acesso em 05 jan 2018]. Disponível em: DOI 10.5151/sma2016-017

9. Nunes TS, Tolfo SR. Assédio moral no trabalho: consequências identificadas por servidores docentes e técnicos administrativos em uma universidade federal brasileira. Revista GUAL. 2012[Acesso 15 dez 2017]; 5(3): 264-86. Disponível em: http://www.redalyc.org/articulo.oa?id=319327516014

10. Paschoal T, Tamayo A. Construção e validação da Escala de Bem Estar no Trabalho. Rev Aval Psic. 2008; 7: 11-22.

11. Tamayo MR, Pinheiro FA, Tróccoli BT, Paz MGT. Construção e validação da Escala de Suporte Organizacional Percebido (ESOP) [Resumo]. Em sociedade Brasileira para o Progresso da Ciência (ORG), $52^{\text {a }}$ Reunião Anual da SBPC, Resumos (CD-Rom). Brasilia: SBPC, 2000.

12. Borsoi ICF. Trabalho e produtivismo: saúde e modo de vida de docentes de instituições públicas de ensino superior. Cader Psic Social Trabalho [Internet] 2012. [Acesso em 10 Jul 2018]; 15(1): 81-100. Disponível em: https://www.revistas.usp.br/cpst/article/view/49623

13. Maués O. A reconfiguração do trabalho docente na educação superior. Educar Rev. [Internet] 2010. [Acesso10 Jul 2018]; 1(esp): 141-160. Disponível em: http://www.scielo.br/pdf/er/nspe1/07.pdf

14. Pereira MAS, Motta EC, Vaz AL, Pinto C, Bernardino O, Melo AC, et al. Sucesso e desenvolvimento psicológico no Ensino Superior: Estratégias de intervenção. Análise Psic. [Internet] 2006. [Acesso 
Maria Lúcia do Carmo Cruz Robazzi, Sergio Valverde Marques dos Santos, Rita de Cássia de Marchi Barcellos Dalri, Vanessa Augusto Bardaquim, Joab Jefferson da Silva Xavier, Luiz Almeida da Silva, Fabiana Cristina Taubert de Freita, Emiliane Moreno Vichnewski

10 Jul 2018]; 1(24):51-9. Disponível em: http://www.scielo.mec.pt/scielo.php?script=sci arttext\&pid $=\mathrm{S} 0870-82312006000100006$

15. Freitas ALP, Souza RGB, Quintella HLM. Qualidade de Vida no Trabalho do técnico-administrativo em IES públicas: uma análise exploratória. Rev Bras Qual vida. [Internet] 2013. [Acesso 06 fev 2018]; 5(2):01-12. Disponível em: https://portaldeinformacao.utfpr.edu.br/Record/oai:periodicos. utfpr:article1382/Description\#tabnav

16. Doneda E, Selow MLC. Motivação ao servidor público. Vitrine Prod Acad. 2016; 4(1): 168-77.

17. Fischer R, Ferreira MC, Assmar EML, Baris G, Berberoglu G, Dalyan, F, et al. Organizational practices across cultures: An exploration in six cultural contexts. Intern Journal Cross Cult Manag. [Internet] 2014. [Acesso em 20 dez 2017]; 14: 105-25. Disponível em: DOI: https://doi. org/10.1177/1470595813510644

18. Dessen MC, Paz MGT. Bem-Estar Pessoal nas Organizações: O Impacto de Configurações de Poder e Características de Personalidade. Psic Teor e Pesq. Brasília. [Internet] 2010. [Acesso em 15 Jan 2018]; 26(3): 549-556. Disponível em: http://www.scielo.br/pdf/ptp/v26n3/a18v26n3.pdf

19. Araújo LMN, Santos VEP, Martins CCFM, Dantas, MSP, Santos, NP, et al. Estresse no cotidiano universitário: estratégias de enfrentamento de docentes da saúde. Rev Fund Care. [Internet] 2016. [Acesso em 20 dez 2017]; 8(4): 4956-64. Disponível em: DOI: http://dx.doi.org/10.9789/2175-5361.2016. v8i4.4956-4964

20. Peterson M, Wilson JF. The culture-work-health model and work stress. American J Health Behavior. [Internet] 2002. [Acesso 20 em dez 2017]; 26:16-24. Disponível em: https://www.ncbi.nlm.nih.gov/ pubmed/11795601

21. Paschoal T, Torres CV, J Porto JB. Felicidade no trabalho: relações com suporte organizacional e suporte social. RAC. [Internet] 2010. [Acesso $10 \mathrm{em}$ jul 2018]; 14(6):1054-72. Disponível em: http:// www.redalyc.org/html/840/84015139005/

22. Caran VCS. Riscos psicossociais e assédio moral no contexto acadêmico. [dissertação]. 2007. Ribeirão Preto: Universidade de São Paulo, Escola de Enfermagem de Ribeirão Preto. [Acesso 10 jul 2018]. Disponível em: http://www.teses.usp.br/teses/disponiveis/22/22132/tde-07012008-134033/

23. Almeida AM. Os transtornos mentais comuns entre estudantes de medicina. Jornal Brasileiro de Psiquiatria. 2007; 56(4):245-251.

24. Silvério KCA, Gonçalves CGO, Penteado RZ, Vieira TPG, Libardi A, Rossi D. Ações em saúde vocal: proposta de melhoria do perfil vocal de professores. Pró-Fono R. Atual. Cient. 2008: 20(3):177-182.

25. Brandão MP, Pimentel FL, Cardoso MF. Impact of academic exposure on health status of university students. Rev Saúd Púb. [Internet] 2011. [Acesso 10 jul 2018]; 45(1): 9-58. Disponível em: https:// www.scielosp.org/scielo.php?script=sci arttext\&pid=S0034-89102011000100006

26. Gabriel AS, Tristão CK, Izar LC, Pina SEM, Franzin LS, Ribeiro DJ, et al. Consumo de álcool e drogas ilícitas entre estudantes de medicina, biologia e enfermagem. Rev Fac Ciên Méd Sorocaba. [Internet] 2004. [Acesso 10 jul 2018]; 6:30-7. Disponível em: https://revistas.pucsp.br/index.php/RFCMS/article/view/311

27. Areias MEQ, Guimarães LAM. Gênero e estresse em trabalhadores de uma universidade pública do Estado de São Paulo. Psic Estudo. [Internet] 2004. [Acesso 10 jul 2018]; 9(2):255-262. Disponível em: http://www.scielo.br/pdf/pe/v9n2/v9n2a11

28. Flores E, Iblin E, Luis, MAV. Uso y actitudes relacionados a las drogas en las estudiantes de enfermería de la Universidad Mayor de San Andrés. Rev Latino-Am Enf [Internet]. 2004[Acesso 10 jul 2018]; 12(spe):376-82. Disponível em: http://www.scielo.br/scielo.php?script=sci arttext\&pid=S010 411692004000700012\&lng=en\&nrm=iso

29. Rodriguez Gazquez MA, Pineda Botero SA, Velez Yepes LF. Características del consumo de tabaco en estudiantes de enfermería de la Universidad de Antioquia (Colombia). Invest y Educ en Enfer [on- 
ISSN-PRINT

$1794-9831$

E-ISSN 2322-7028

Vol. 16 No. 2

May - Ago 2019

Cúcuta, Colombia line]. 2010 [Acesso 10 jul 2018]; 28(3):370-383. Disponível em: http://bibliotecadigital.udea.edu.co/ handle/10495/5123

30. Loiola ESC, Alves HMC, Siqueira MMM. Relação entre percepção de justiça e bem-estar no trabalho em profissionais brasileiros. Rev Psic Saúde e Doenças. [Internet] 2017. [Acesso em 10 jan 2018]; 18(1): 85-90. Disponível em: http://www.redalyc.org/pdf/362/36250481007.pdf 